\title{
EVALUACIÓN DE PANEL SNP EN GENES CANDIDATOS DE VÍAS METABÓLICAS PARA CARNE EN HEREFORD
}

\author{
EVALUATION OF PANEL SNP IN METABOLIC PATHWAYS CANDIDATE GENES \\ FOR MEAT IN HEREFORD
}

\author{
Branda Sica, A. ${ }^{1^{*}}$; Ravagnolo, O.2; Brito, G. ${ }^{2}$; Baldi, F.2; LaManna, A. ${ }^{2}$; Banchero, G. ${ }^{2}$; \\ Navajas, E.A. ${ }^{1}$; Rincón, G. ${ }^{3}$ y Medrano, J.F. ${ }^{3}$
}

\begin{abstract}
${ }^{1}$ Instituto Nacional de Investigación Agropecuaria. Unidad de Biotecnología. Rincón del Colorado. Uruguay. *abranda@inia.org.uy

${ }^{2}$ Instituto Nacional de Investigación Agropecuaria. Programa Nacional de Carne y Lana. Tacuarembó. Uruguay.

${ }^{3}$ Departamento de Ciencia Animal. Universidad de California. Davis. California. USA.
\end{abstract}

\section{Palabras CLAVE ADICIONALES}

Bovinos de carne. Composición de canal. Calidad de carne. Perfil de ácidos grasos.

\section{RESUMEN}

Se realizó una validación preliminar de un panel de marcadores SNP seleccionados de genes candidatos, relacionados al eje endócrino que regulan la hormona de crecimiento GH/IGF1, y a la formación y desaturación de ácidos grasos SCD/ SREBP1, con características de composición de canal, calidad de carne y perfil de ácidos grasos de la grasa intramuscular en novillos Hereford sometidos a diferentes tratamientos de alimentación durante la etapa de recría y engorde intensivo. Se ajustaron las variables por el efecto de los 8 tratamientos nutricionales y se calcularon los valores residuales para los análisis de asociación con los marcadores SNP. Se determinó un total de 11696 genotipos de 237 novillos utilizando un panel de 58 tag-SNP en los genes GHR, IGF1, IGFBP6, PMCH, SOCS2, STAT6, SCD1, SCD5, SREBP1, SCAP, INSIG1, INSIG2, MBTPS1, MBTPS2 y SRPR, de los cuales se encontraron 11 SNP polimórficos que estuvieron asociados significativamente: GHR_ rs41639262 sobre EGSu y MARBL; IGF1_rs136251088 sobre PCC, PCE y PISTOLA; PMCH_rs135033882 sobre OMEGA36; SOCS2_ss252841026 sobre Terneza2d; STAT6_rs109171041 sobre Terneza2d; STAT6_ rs110097583 sobre Terneza2d; INSIG1_ rs109314460 sobre Terneza21d; INSIG1_ ss252452220 sobre OMEGA36; SCD5_

\section{AdDitionAL KEYWORDS}

Beef cattle. Carcass composition. Meat quality. Fatty acid profile.

ss252452202 sobre PUFA y Terneza2d; SCD5 rs43687655 sobre EGSu y OMEGA36; SRPR rs42178091 sobre GRASAINTR, SFA, MUFA, PCC, PCE y PISTOLA. Estudios adicionales podrían confirmar estos efectos, posibilitando mejorar la calidad de la canal y de la carne, de animales sometidos a diferentes tratamientos de alimentación a través de la selección.

\section{SUMMARY}

The phenotype-genotype associations of a panel of SNP markers selected from candidate genes related to the growth hormone $\mathrm{GH} /$ IGF1endocrine axis and the fatty acid synthesis and desaturation SCD/SREBP1 pathways, were examined. Phenotypes of interest were carcass composition, meat quality and fatty acid profiles of intramuscular fat in Hereford steers under different feeding treatments during the growth and finishing stages. Variables were adjusted for the effect of eight nutritional treatments and residual values were used in the association analysis with the SNP markers. A total of 11696 genotypes of 237 steers were determined using a panel of 58 tagSNP in the GHR, IGF1, IGFBP6, PMCH, SOCS2, STAT6, SCD1, SCD5, SREBP1, SCAP, INSIG1, INSIG2, MBTPS1, MBTPS2 and SRPR genes. Atotal 
of 11 polymorphic SNP were significantly associated with traits of interest: GHR_rs41639262 on EGSu and MARBL; IGF1_rs136251088 on PCC, PCE and PISTOLA; PMCH_rs135033882 on OMEGA36; SOCS2_ss252841026 on Terneza2d; STAT6_ rs109171041 on Terneza2d; STAT6 rs110097583 on Terneza2d; INSIG1_rs109314460 on Terneza21d; INSIG1_ss252452220 on OMEGA36; SCD5_ss252452202 on PUFA and Terneza2d; SCD5_rs43687655 on EGSu and OMEGA36; SRPR_rs42178091 on GRASAINTR, SFA, MUFA, PCC, PCE and PISTOLA. Additional studies could validate the marker-trait association effects that would make it possible to use these genetic markers to improve carcass and meat quality of animals under different feeding regimes.

\section{INTRODUCCIÓN}

La producción de carne bovina en Uruguay representa una importante fuente de divisas, siendo la calidad de la canal y de la carne elementos clave para su competitividad. La composición de canal, calidad de la carne y perfil de ácidos grasos de la grasa intramuscular son factores de interés económico y de exigencia por parte de los mercados y consumidores. El nivel de terminación de un animal depende de su tamaño corporal y, a su vez, es influenciado por la dieta utilizada durante el engorde. Los animales con engorde intensivo durante la recría tendrán mayor nivel de engrasamiento en la canal (Baldi et al., 2010). El uso de mutaciones funcionales como marcadores que explican una proporción significativa de la varianza fenotípica es la opción más eficaz para la selección asistida por marcadores si la estimación de los efectos está apropiadamente validada (Dekkers, 2004).

La hormona de crecimiento (GH) está implicada en varios procesos metabólicos y fisiológicos (Etherton, 2004; Chagas et al., 2007); para producir sus efectos debe unirse a su receptor (GHR) (Edens y Talamantes, 1998; Zhu et al., 2001). El gen GHR bovino está en un QTL (loci de rasgos complejos o cuantitativos, siglas en inglés) para producción y composición de leche, y porcen- taje de grasa corporal en el cromosoma 20 (Georges et al., 1995; Arranz et al., 1998; Zhang et al., 1998). Ge et al. (2003) sugirieron que los polimorfismos en el gen GHR podrían ser marcadores potenciales para las concentraciones séricas de IGF1 (factor de crecimiento para la insulina) en ganado Angus. Los trabajos de Rincón et al. (2009) y DeAtley et al. (2010) han sugerido que el gen STAT6 (transductor de señal y activador de transcripción 6) es un gen candidato posicional en el cromosoma bovino 5 para características de crecimiento y canal en el ganado bovino. Las proteínas STAT están implicadas en la señalización celular mediante cascadas en citoquinas o hormonas, tales como GH y leptina, que se unen a sus receptores (Rawlings et al., 2004; Fruhbeck, 2006; Schindler y Plumlee, 2008).

El conocimiento de la calidad nutricional y composición de ácidos grasos saturados, mono y poli-insaturados en la carne es de gran importancia en relación con el nivel de colesterol en la sangre cuyo aumento, favorece la arteriosclerosis y enfermedades coronarias en los humanos (Kromhout et al., 2002). Estos efectos son debidos principalmente al ácido láurico (C12:0), el ácido mirístico (C14:0) y elácido palmítico (C16:0), mientras que los otros ácidos grasos saturados (C4:0 a C10:0 y C18:0) son neutrales (Bonanome y Grundy, 1988).

Existen estudios que argumentan el control genético de la variación de la formación y desaturación de ácidos grasos en bovinos de carne (Taniguchi et al., 2004; Orrù et al., 2011). La enzima delta-9-desaturasa (SCD) había sido propuesta como un nuevo regulador de la homeostasis energética corporal (Paton y Ntambi, 2009) y es uno de los genes candidatos más prometedores para explicar la variabilidad de la composición y perfil de ácidos grasos. Esta enzima, localizada en el retículo endoplásmico, es responsable en la catálisis de la inserción de un enlace doble en la posición delta, para la producción de cis-9-trans-11 ácido linoleico conjugado (CLA) (Bauman et al., 1999; Schmid et al., 
2006) en la grasa de rumiantes, y para la síntesis deácido palmítoleico (C16:1) y oleico (C18:1, cis-9) (Enoch et al., 1976). Convierte los ácidos grasos saturados (SFA) en ácidos mono-insaturados (MUFA) para producir CLA. Se han caracterizado dos isoformas diferentes de SCD: SCD1 y SCD5, localizadas en los cromosomas bovinos 26 y 6, respectivamente (Campbell et al., 2001; Lengi y Corl, 2007). La expresión de SCD está regulada por varios factores de transcripción, proteasas y otros elementos reguladores incluyendo SREBP1 (proteína 1 de unión al elemento regulador del esterol), INSIG (proteína inducida por la insulina) y SCAP (clivaje de activación por la proteína SREBP), entre otros (Mauvoisin y Mounier, 2011). Jacobs et al. (2011), evaluaron el patrón de expresión de SCD5 en la glándula mamaria, sugiriendo que este gen podría estar regulado diferencialmente con respecto a SCD1 por factores dietéticos. No se ha encontrado ningún trabajo que detalle la expresión de SCD5 en tejido adiposo o muscular, además de ser altamente expresado en el cerebro (Lengi y Corl, 2007).

El objetivo de este trabajo fue la validación preliminar de un panel de marcadores SNP (polimorfismo de nucleótido simple, siglas en inglés) seleccionados de genes candidatos, relacionados al eje endócrino que regulan la hormona de crecimiento GH/ IGF1, y la formación y desaturación de ácidos grasos SCD/SREBP1, con las características de carcasa, calidad de la carne y perfil de ácidos grasos de la grasa intramuscular en novillos Hereford sometidos a diferentes manejos nutricionales durante la etapa de recría y engorde intensivo.

\section{MATERIAL Y MÉTODOS}

\section{ANIMALES Y DATOS FENOTÍPICOS}

Para realizar el análisis de asociación entre los marcadores SNP desarrollados por Rincón et al. (2009, 2012) y las características de composición de canal, calidad de carne y perfil de ácidos grasos, se emplea- ron 237 novillos Hereford, recríados y engordados en la Unidad del Lago de INIA La Estanzuela, Colonia, desde junio del 2008 (invierno) hasta marzo del 2009 (otoño) y que corresponden a una investigación sobre el efecto de niveles nutricionales durante el post-destete y período de terminación (Baldi et al., 2010). Se realizaron dos manejos nutricionales contrastantes (corral versus pasturas) al destete de los terneros (170 kg), asignándolos a uno de los 4 diferentes tratamientos (corral concentrado alto, CA; corral concentrado bajo, CB; pastura alto, PA y pastura bajo, PB), luego durante la primavera y verano (septiembre del 2008 hasta febrero del 2009) se juntaron a un manejo conjunto sobre praderas permanentes. En la etapa de terminación de los animales (entre 350-360 kg), en los últimos 90-180 días, se volvieron a subdividir en forma aleatoria en pasturas de alta calidad o en confinamiento. Se faenaron los animales cuando alcanzaron los $500 \mathrm{~kg}$. En resumen, se han generado 8 tratamientos de alimentación de la forma como se describe: T1: CA + Corral; T2: CB + Corral; T3: PA + Corral; T4: PB + Corral; T5: CA + Pastura; T6: CB + Pastura; T7: PA + Pastura; y T8: PB + Pastura.

Los novillos utilizados provenían de un solo origen y estuvieron sujetos a condiciones experimentales desde el post-destete hasta el momento de la faena y cuentan con toda la información recolectada en el transcurso del ensayo.

Para este trabajo se consideraron las mediciones del área de ojo de bife (AOB$\mathrm{cm}^{2}$ ) y el espesor de grasa subcutánea por ultrasonido (EGSu-mm) entre la $12-13^{\mathrm{a}}$ costilla en el animal vivo y el peso de la canal caliente (PCC-kg) en el frigorífico. Posterior al enfriado se registró el peso de la canal (PCE-kg) y el pH a las 48 horas post-faena (pH2d) en el músculo longissimus thoracis entre la $12^{\mathrm{a}}$ y $13^{\mathrm{a}}$ costilla y después del cuarteo se tomó el peso del corte pistola (PISTOLA-kg), que se obtiene a partir de la media res seccionando transversalmente el 
raquis entre la 10 y $11^{\mathrm{a}}$ vertebra dorsales; es la pieza que comprende la totalidad del cuarto trasero a 3 costillas y está constituido por los siguientes cortes: bife angosto, lomo, cuadril, colita de cuadril, peceto, cuadrada, nalga de adentro, bola de lomo, tortuguita y garrón. Al desosado se pesaron los principales cortes provenientes de PISTOLA (lomo, bife angosto y cuadril -rump and loin, RL-kg), los recortes de carne y grasa y los huesos. Se extrajeron muestras del músculo longissimus lumborum, las cuales fueron maduradas durante 2 (Terneza2d-kgF) y 21 días (Terneza21d-kgF) a $2-3{ }^{\circ} \mathrm{C}$ para la determinación del grado de dureza (fuerza de corte de una muestra de carne -bife angosto-, Warner-Braztler) en forma objetiva. La cantidad de veteado ó marmoleado (cantidad y distribución de la grasa intramuscular) se estimó de manera subjetiva mediante un puntaje según la escala del USDA (Departamento de Agricultura de EE.UU). Se analizó químicamen- te el porcentaje de grasa intramuscular (GRASAINTR-\%), a través del método de Folch et al.(1957), y se determinó el perfil de ácidos grasos por cromatografía de gases: porcentaje de ácidos grasos saturados (FSA\%), ácidos mono-insaturados (MUFA-\%), ácidos poli-insaturados (PUFA-\%), y la relación ácidos poli-insaturados n-3 y n-6 (OMEGA36-relación n3/n6). Las estadísticas descriptivas con sus valores de media, desvío estándar, mínimo y máximo para las variables de composición de carcasa, calidad de carne y perfil de ácidos grasos, se muestran en la tabla I.

\section{AnÁlisis Estadístico}

Todas las variables analizadas fueron previamente ajustadas por los 8 tratamientos nutricionales utilizados. La estimación de los efectos de los SNP se realizó a partir de los residuales obtenidos, no pudiéndose analizar ajustes por el efecto padre por ausencia de información genealógica. El mo-

Tabla I. Estadística descriptiva de las características de composición de canal, calidad de carne y perfil de ácidos grasos en engorde de novillos Hereford. (Descriptive statistics of carcass composition, meat quality and fatty acid profiles of Hereford steers).

\begin{tabular}{lccccc}
\hline Característica & $\mathrm{n}$ & Media & Desvío estándar & Mínimo & Máximo \\
\hline EGSu-mm $^{2}$ & 226 & 7,483 & 2,114 & 4,064 & 14,224 \\
AOB-cm $^{2}$ & 227 & 54,467 & 5,005 & 42,559 & 71,233 \\
PCC-kg & 227 & 249,865 & 19,269 & 201,600 & 302,000 \\
PCE-kg & 227 & 243,072 & 18,621 & 197,000 & 292,000 \\
PISTOLA-kg & 227 & 53,599 & 4,360 & 42,336 & 67,160 \\
RL-kg & 225 & 11,842 & 1,056 & 8,750 & 15,200 \\
Terneza2d-kgF & 221 & 4,016 & 1,491 & 1,840 & 10,205 \\
Terneza21d-kgF & 220 & 3,328 & 0,831 & 2,015 & 8,300 \\
pH2d & 221 & 5,517 & 0,138 & 5,350 & 6,520 \\
MARBL-escala USDA & 225 & 224,711 & 75,473 & 100,000 & 400,000 \\
GRASAINTR-\% & 225 & 3,37 & 1,17 & 1,48 & 8,4 \\
SFA-\% & 217 & 45,041 & 2,341 & 37,350 & 52,710 \\
MUFA-\% & 217 & 46,995 & 2,876 & 39,110 & 55,190 \\
PUFA-\% & 217 & 7,514 & 2,080 & 4,060 & 16,280 \\
OMEGA 36-relación n3/n6 & 217 & 0,431 & 0,207 & 0,181 & 1,839 \\
\hline
\end{tabular}

${ }^{1}$ Niveles de marmoleado según escala USDA: 100-199= prácticamente desprovisto; 200-299= trazas; $300-399=$ leve; 400-499= poco. 
delo utilizado para la generación de los residuales fue: $\mathrm{y}_{\mathrm{ij}}=\mathrm{T} 8_{\mathrm{i}}+\mathrm{e}_{\mathrm{ij}}$; donde $\mathrm{y}_{\mathrm{ij}}$ es la característica analizada del i-ésimo animal en el j-ésimo tratamiento; $\mathrm{T} 8$ es el efecto de la i-ésima clase del Tratamiento (i de 1 a 8); $\mathrm{e}_{\mathrm{ij}}$ es el residual. Estos análisis fueron realizados usado el procedimiento GLM del paquete estadístico SAS (SAS, 2007).

\section{EXTRACCIÓNDEADNYGENOTIPADO}

A partir de las muestras de sangre de 237 novillos Hereford, se realizó la extracción del ADN de acuerdo con el protocolo de Sambrock et al. (1989). Para el genotipado en estos novillos se utilizó un panel de SNP desarrollado por Rincón et al. (2009, 2012), con el fin de explicar las variaciones a nivel de crecimiento, rendimiento carnicero y calidad de la carcasa. Dicho panel consistió en 58 SNPs en genes candidatos relacionados con el eje que regula la hormona de crecimiento GH/IGF1: GHR, IGF1, IGFBP6 (proteína 6 de enlace del IGF), PMCH (hormona concentradora de pro-melanina), SOCS2 (supresor de señal de las citoquinas) y STAT6, y con la formación y desaturación de ácidos grasos SCD/SREBP1: SCD1, SCD5, SREBP1, SCAP, INSIG1, INSIG2, MBTPS1 (peptidasa del factor de transcripción de membrana, sitio 1), MBTPS2 (peptidasa del factor de transcripción de membrana, sitio 2) y SRPR (receptor de reconocimiento de señal de partículas -proteína de acoplamiento). Estos marcadores analizados representan regiones en desequilibrio de ligamiento para diferentes genes que fueron resecuenciados en diferentes razas bovinas (tag-SNP). Las designaciones de los SNP fueron informadas previamente por Rincón et al. (2009, 2012) y se realizaron de acuerdo a la identificación de cada uno de ellos está detallada en el dbSNP del NCBI (http://www.ncbi.nlm.nih.gov/ $\mathrm{SNP} /$; accedido por última vez el 4 de enero, 2013). El genotipado fue llevado a cabo utilizando la plataforma Sequenom MassARRAY®, en GeneSeek Inc. (Lincoln, NE, USA).
ANÁLISISDELOSSNP, ASOCIACIÓN Y PROCESAMIENTO DEDATOS

Para los análisis de los SNP se utilizó el programa HelixTree SVS versión 7 (Golden Helix Inc., Bozeman, Montana, USA). Se calcularon sus frecuencias del alelo menor (MAF), proporción de individuos genotipados (call rate) y valor p del test en equilibrio de Hardy-Weinberg (HWE p) para cada uno de los SNP. Se realizó el análisis de asociación genotípica con módulo de análisis genético (modelo aditivo) y regresión del HelixTree SVS7. Se han utilizado los criterios de calidad estándar en este trabajo: se han eliminado genotipos que presentaron call rate inferior al 80 \% y MAF igual ó inferior a 0,01 . Se analizaron las estimaciones del efecto de sustitución alélica por regresión del fenotipo sobre las variables del genotipo.

\section{RESULTADOSYDISCUSIÓN}

Se obtuvo un total de 11696 genotipos en 237 novillos Hereford. De los 58 SNP originales del panel se descartaron 18 SNP, de los cuales 2 no se pudieron identificar correctamente en ninguna de las muestras, 7 eran sólo monomórficos, y 9 tenían call rate inferior al $80 \%$ y MAF igual o menor a 0,01 . Esto se debe a que la raza Hereford utilizada no estaba incluida en el panel de SNP en donde se detectaron los SNP. Por tanto, quedaron 40 tag-SNP identificados en los genes de las vías GH/IGF1 y SCD/ SREBP1 para analizar sus efectos sobre las características previamente corregidas por el efecto de los 8 tratamientos durante la etapa de recría y engorde intensivo de novillos. Se encontraron 11 SNP polimórficos en los genes IGF1, GHR, PMCH, SOCS2, STAT6, INSIG1, SCD5 y SRPR que estuvieron asociados significativamente $(\mathrm{p}<0,05)$. La tabla II resume la estadística de $\operatorname{tag}$-SNP significativos utilizados en este estudio, los marcadores con la identificación de los SNP en dbSNP del NCBI, MAF, call rate, y test de HWE-p en los novillos Hereford de 
este estudio. La tabla III muestra los efectos de sustitución alélica (ESA) y las medias dependientes de los genotipos con los marcadores significativos $(\mathrm{p}<0,05)$ para las características en engorde de novillos. Los demás polimorfismos analizados en los genes de las vías GH/IGF1 y SCD/SREBP1 no fueron significativos para AOB, RL y $\mathrm{pH} 2 \mathrm{~d}$.

Los valores de ESA mostraron que los animales seleccionados con genotipo AA para GHR_rs41639262 tienen menos puntuación (escala) de marmoleado y espesor de grasa subcutánea (menos de 20 y 350 mm, respectivamente) que los de genotipo GG más abundante (tabla III). El alelo A del gen GHR está asociado con una disminución en la disposición de marmoleado y espesor de grasa subcutánea. Este SNP del gen GHR se localiza en la región reguladora del promotor y puede afectar la función del gen. Las variaciones en la secuencia pueden clasificarse como funcional y no funcional. El SNP funcional afecta el producto del gen, mientras que el SNP no funcional está relacionado con las regiones funcionales del gen (Khan et al., 2006; Ron y Weller, 2007). Este estudio coincide con los resultados obtenidos por Hale et al. (2000) que utilizaron el microsatélite GT en el promotor del gen descrito por Lucy et al. (1998) que contenía una versión corta del microsatélite en novillos Angus con menos peso al destete, peso de la canal y puntuación (ó escala) de marmoleado en relación a los novillos con el alelo más largo. Baeza et al. (2011) encontraron un efecto significativo sobre grasa intramuscular, donde la distribución de las frecuencias de los marcadores GHR en novillos Brangus mostró que el genotipo más abundante del SNP del promotor de este gen no fue favorable para la disposición de veteado (o marmoleado) mientras que el genotipo más abundante del otro SNP del gen GHR favoreció el mayor contenido de grasa. Este SNP fue identificado previamente por Ge et al. (1999) y analizado para las asociaciones con características de crecimiento con niveles séricos de IGF1 en sangre (Ge et al., 2003). También se evaluó para las asociaciones con características de producción de carne (Maj et al., 2004, 2006).

Tabla II. Estadística de tag-SNP significativos $(p<0,05)$ utilizados para los análisis de asociación en engorde de novillos Hereford. (Statistics of significant tag- SNP used for association analysis in finishing of Hereford steers).

\begin{tabular}{|c|c|c|c|c|}
\hline Marcador (Gen/ NCBI ID) ${ }^{1}$ & Call rate & Alelo menor & MAF & HWEP \\
\hline IGF1_rs136251088 & 0,93 & $\mathrm{~T}$ & 0,262 & $<0,001$ \\
\hline GHR rs41639262 & 0,96 & G & 0,348 & 0,452 \\
\hline PMCH_rs135033882 & 0,81 & $\mathrm{~T}$ & 0,381 & 0,298 \\
\hline socs $2 \overline{2}$ ss252841026 & 0,81 & $\mathrm{~T}$ & 0,351 & 0,455 \\
\hline STAT6_rs109171041 & 0,97 & $\mathrm{C}$ & 0,402 & 0,588 \\
\hline STAT6_rs110097583 & 0,97 & $A$ & 0,350 & 0,246 \\
\hline INSIG1_rs109314460 & 0,96 & G & 0,115 & 0,475 \\
\hline INSIG1_ss252452220 & 0,97 & $\mathrm{~T}$ & 0,120 & 0,114 \\
\hline SCD5 ss252452202 & 0,97 & C & 0,311 & 0,154 \\
\hline SCD5 rs43687655 & 0,97 & $\mathrm{~T}$ & 0,5 & 0,217 \\
\hline SRPR rs42178091 & 0,97 & $\mathrm{C}$ & 0,219 & 0,546 \\
\hline
\end{tabular}

${ }^{1}$ El marcador designado es el gen seguido con la identificación dbSNP en NCBI, posición del SNP de acuerdo al Btau_4.6.1 partir del ensamblado genómico (http://www.ncbi.nlm.nih.gov/SNP/; accedido por última vez el 04/01/2013).

Archivos de zootecnia vol. 63, núm. 241, p. 78. 
Garret et al. (2008) analizaron los polimorfismos de la secuencia en la región reguladora del promotor $(1,195 \mathrm{pb})$, que consistió de 9 SNP y un microsatélite. Tres de estos SNP fueron descritos previamente por Hale et al. (2000) y 1 SNP por Sherman et al. (2008). Los análisis de re-secuenciación revelaron diferencias en la región promotora de GHR entre ganado Brahman (Bos indicus) y razas de Bos taurus (Garret et al., 2008). Sin embargo, el promotor del gen GHR bovino es muy complejo y requiere de investigaciones adicionales para determinar la funcionalidad de los genes, si este SNP es una mutación casual. En el trabajo de Baldi et al. (2010), se observó que los animales, los grupos CA y CB, presentaron mayor cobertura de grasa en la canal cuando la terminación fue a corral. Con este marcador del gen GHR se podría seleccionar aquellos animales que posean menor nivel de engrasamiento y por ende carne más magra, cualidades que forman parte de las exigencias del mercador consumidor.

Los otros 5 SNP en los genes IGF1, PMCH, SOCS2 y STAT6 del eje endócrino GH/IGF1 están localizados en el cromosoma bovino 5. En el caso de IGF1_rs136251088 para peso de la canal caliente y enfriada, se observó que el promedio de los genotipos del heterocigota GT $(-2,042 \mathrm{y}-2,114)$ fueron similares al homogicota TT $(2,604$ y 2,678$)$ y diferentes al heterocigota GG (-11,185 y -10,983) (tabla III). Esta observación podría indicar que no hubo un ajuste del modelo aditivo a pesar que los valores coincidieron con los de regresión con un número menor de animales con genotipo GG. Sin embargo, los valores de ESA para este SNP del gen IGF1 mostraron que los animales seleccionados con genotipo GG tienen menos peso de canal caliente y enfriada (5 kg menos), y de pistola (1 kg menos) que los de genotipo TT (tabla III). El alelo G del gen IGF1 está asociado con una disminución en el peso de la canal caliente y enfriada y de la pistola. Este SNP del gen IGF1 está localizado en el intrón 4 y no afecta la secuencia proteica.
Para PMCH_rs135033882 en la relación de ácidos poli-insaturados n-3 y n-6, también se observó que el modelo aditivo no se ajusta al promedio de los genotipos, y podría asumirse otro modelo de acción génica, ya que se mostró un aumento de $23 \%$ en la relación de ácidos poli-insaturados n-3 y n6 para los animales seleccionados con genotipo AA que los de genotipo TT (tabla III). Este SNP del gen PMCH es una sustitución sinónima localizada en la región no traducida 5' (UTR 5'). Los polimorfismos en las regiones UTR 5' y 3' de un gen pueden afectar un producto génico alterando la unión del factor de transcripción (Crawford y Nickerson, 2005).

Los animales seleccionados con genotipo CC para SOCS2_ss252841026 tienen menos terneza en la carne con 2 días de maduración que los de genotipo TT (tabla III). El alelo C del gen SOCS2 está asociado con una disminución en la terneza de la carne con 2 días de maduración. Este SNP del gen SOCS2 también es una sustitución sinónima localizada en la UTR 5'.

Los 2 SNP del gen STAT6 (STAT6_ rs109171041 y STAT6_rs110097583) son sinónimos y están situados en el intrón 1 y 8. Los valores de ESA para STAT6_ rs109171041 mostraron una diferencia en los promedios de los genotipos observados para la terneza de la carne con maduración de 2 días que aumentó $(-0,216 \mathrm{kgF})$ en los animales seleccionados con genotipo GG con respecto a los de genotipo CC (tabla III). En cambio, los animales seleccionados con genotipo GG para STAT6_rs110097583 tienen menos terneza en la carne con 2 días de maduración que aquellos con genotipo AA (tabla III). Estos resultados concuerdan con los obtenidos por Rincón et al. (2009) que encontró polimorfismos en este gen que están asociados con características de la canal y de crecimiento en ganado de engorde en condiciones de confinamiento (feedlot). El gen STAT6 actúa de mediador en la señalización y funciona como un factor de transcripción. Este gen integra el 
complejo STAT que está conformado por diferentes proteínas que participan en la activación de procesos enzimáticos requeridos para la síntesis del IGF1 (Schindler y Plumlee, 2008). El gen SOCS incluye una familia de 8 proteínas que se asocian negativamente al IGF1 inhibiendo su secreción (Cooke et al., 2008).

Debido a la proximidad de los genes en el cromosoma bovino 5 se mostró que afectan los niveles de grasa, marmoleado y terneza de la carne en bovinos de carne, es posible que los SNP en los genes PMCH y SOCS2 interactuando a través de sus factores de transcripción con otro gen casual, en ese caso con el promotor del GHR, producen menos grasa y carne menos tierna.

Los 2 SNP del gen INSIG1 (INSIG1_ rs109314460 e INSIG1_ss252452220) están localizados en el cromosoma bovino 4 y se encuentran en los exones 1 y 4, respectivamente. Estas mutaciones no sinónimas (funcionales) afectan la secuencia de proteína por el cambio de aminoácidos de Ser/Gly para INSIG1_rs109314460 y de Leu/Phe para INSIG1_ss252452220 (Rincón et al., 2012). Los valores de ESA mostraron que los animales seleccionados con genotipo AA para INSIG1_rs109314460, tienen más terneza en la carne con 21 días de maduración que los de genotipo GG (tabla III). Los animales seleccionados con genotipo CC para INSIG1_ss252452220 tienen 35\% más en la relación de ácidos poli-insaturados n3 y n-6 que los de genotipo TT (tabla III). Como estos 2 SNP fueron analizados en un número muy reducido habría que confirmar si es una mutación funcional sobre la terneza en la carne con 21 días de maduración y la relación de ácidos poli-insaturados n-3 y n-6.

Los 2 SNP en el gen SCD5 (SCD5 ss252452202 y SCD5_rs43687655) localizados en el cromosoma bovino 6 (en el exón 3 y 4 , respectivamente) son mutaciones sinónimas y no afectan la conformación proteica. Los animales seleccionados con genotipo TT para SCD5_ss252452202 tie- nen 52 \% más de ácidos poli-insaturados y menos terneza con 2 días de maduración que los de genotipo CC (tabla III). El alelo C del gen SCD5 está asociado con un aumento de porcentaje de ácidos grasos poliinsaturados y una disminución en la terneza de la carne con 2 días de maduración. Este gen está implicado en la biosíntesis de ácidos grasos no saturados y está relacionado con la diferenciación de adipositos maduros del tejido adiposo intramuscular durante la fase temprana del crecimiento (Lengi y Corl, 2010). Para SCD5_ rs43687655, los animales seleccionados con genotipo CC tienen $330 \mathrm{~mm}$ menos de espesor de grasa subcutánea y 17\% menos en la relación de ácidos poli-insaturados n-3 y n-6 que los de genotipo TT (tabla III). El alelo $\mathrm{C}$ del gen SCD5 está asociado con una disminución en espesor de grasa subcutánea y en la relación de ácidos poli-insaturados n-3 y n6. Sin embargo, esos 2 SNP del gen SCD5 son mutaciones sinónimas, la sustitución ocurre en una región funcional y la información será transcrita en ARNm.

Para SRPR_rs42178091, los animales seleccionados con genotipo AA tienen más ácidos grasos saturados y grasa intramuscular, menos ácidos monoinsaturados, peso de la canal caliente y enfriada, y de pistola que los de genotipo TT (tabla III). El alelo A del gen SRPR está asociado con un aumento en el porcentaje de ácidos grasos saturados y de grasa intramuscular, una disminución en el porcentaje de ácidos mono-insaturados, y en el peso de la canal caliente y enfriada, y de la pistola. Este SNP está localizado en el cromosoma bovino 29 y se encuentra en una región no codificante. Baldi et al. (2010) encontraron efectos significativos del manejo nutricional durante la recría sobre el peso de la canal, cuando la terminación fue realizada en condiciones de engorde a corral. El grupo PB fue el que presentó los menores pesos de canal caliente en relación al grupo CA. Al tener menor contenido de ácidos grasos mono-insaturados (principal- 
Tabla III. Efectos y medias dependientes de los genotipos con los marcadores significativos $(p<0,05)$ para las características en engorde de novillos Hereford. (Effects and genotype averages for significant genetic markers for Hereford steer traits).

\begin{tabular}{|c|c|c|c|c|c|c|c|}
\hline \multirow{2}{*}{$\begin{array}{l}\begin{array}{l}\text { Marcador } \\
(\text { Gen/NCBIID) }\end{array} \\
\text { IGF1 rs136251088 }\end{array}$} & \multirow[t]{2}{*}{ Característica } & \multirow[t]{2}{*}{$\mathrm{n}$} & \multicolumn{3}{|c|}{$\begin{array}{l}\text { Medias dependientes } \\
\text { de genotipos }\end{array}$} & \multirow[b]{2}{*}{$E S A$} & \multirow[b]{2}{*}{ SESA } \\
\hline & & & GG & $G T$ & $T T$ & & \\
\hline & $\mathrm{PCC}(\mathrm{kg})$ & 227 & $-11,185$ & $-2,042$ & 2,604 & $-4,880$ & 0,044 \\
\hline & PCE (kg) & 227 & $-10,983$ & $-2,114$ & 2,678 & $-5,005$ & 0,034 \\
\hline & PISTOLA (kg) & 227 & $-2,007$ & $-0,509$ & 0,611 & $-1,140$ & 0,026 \\
\hline \multirow[t]{3}{*}{ GHR rs41639262 } & & & $A A$ & $A G$ & GG & & \\
\hline & EGSu (mm) & 234 & $-0,473$ & $-0,110$ & 0,226 & $-0,346$ & 0,045 \\
\hline & MARBL (escala USDA) & 234 & $-26,879$ & $-5,855$ & 14,050 & $-20,317$ & 0,003 \\
\hline \multirow[t]{2}{*}{ PMCH rs135033882 } & & & $A A$ & AT & $T T$ & & \\
\hline & OMEGA36 (relación n3/n6) & 197 & 0,05 & $-0,007$ & $-0,008$ & 0,023 & 0,027 \\
\hline \multirow[t]{2}{*}{ socs2 ss252841026 } & & & $C C$ & $C T$ & $T T$ & & \\
\hline & Terneza2d (kgF) & 198 & 0,561 & $-0,019$ & $-0,190$ & 0,311 & 0,009 \\
\hline \multirow[t]{2}{*}{ STAT6 rs109171041 } & & & $G G$ & GC & $C C$ & & \\
\hline & Terneza2d (kgF) & 235 & $-0,412$ & 0,071 & 0,093 & $-0,216$ & 0,031 \\
\hline \multirow[t]{2}{*}{ STAT6 rs110097583 } & & & GG & $G A$ & $A A$ & & \\
\hline & Terneza2d (kgF) & 237 & 0,565 & 0,048 & $-0,204$ & 0,341 & 0,001 \\
\hline \multirow[t]{2}{*}{ INSIG1 rs109314460 } & & & $A A$ & $A G$ & $G G$ & & \\
\hline & Terneza21d (kgF) & 234 & $-0,567$ & $-0,243$ & 0,081 & $-0,323$ & 0,003 \\
\hline \multirow{2}{*}{ INSIG1 ss252452220 } & & & $C C$ & $C T$ & $T T$ & & \\
\hline & OMEGA36 (relación n3/n6) & 237 & 0,161 & $-0,004$ & $-0,005$ & 0,035 & 0,007 \\
\hline \multirow[t]{3}{*}{ SCD5 Ss252452202 } & & & $T T$ & $T C$ & $C C$ & & \\
\hline & PUFA (\%) & 235 & 1,132 & 0,076 & $-0,245$ & 0,520 & 0,016 \\
\hline & Terneza2d (kgF) & 235 & 0,584 & 0,003 & $-0,119$ & 0,245 & 0,034 \\
\hline \multirow[t]{3}{*}{ SCD5 rs43687655 } & & & $C C$ & $C T$ & $T T$ & & \\
\hline & EGSu (mm) & 237 & $-0,294$ & $-0,105$ & 0,357 & $-0,329$ & 0,044 \\
\hline & OMEGA36 (relación n3/n6) & 237 & $-0,010$ & $-0,009$ & 0,024 & $-0,017$ & 0,044 \\
\hline \multirow[t]{7}{*}{ SRPR rs42178091 } & & & $A A$ & $A C$ & $C C$ & & \\
\hline & SFA (\%) & 237 & 0,663 & 0,386 & $-0,266$ & 0,565 & 0,019 \\
\hline & GRASAINTR (\%) & 237 & 0,115 & $-0,004$ & $-0,008$ & 0,032 & 0,004 \\
\hline & MUFA (\%) & 237 & $-1,292$ & $-0,374$ & 0,285 & $-0,719$ & 0,005 \\
\hline & PCC (kg) & 237 & $-7,564$ & $-2,925$ & 1,856 & $-4,747$ & 0,022 \\
\hline & PCE (kg) & 237 & $-7,692$ & $-2,993$ & 1,912 & $-4,856$ & 0,016 \\
\hline & PISTOLA (kg) & 237 & $-1,570$ & $-0,520$ & 0,324 & $-0,893$ & 0,043 \\
\hline
\end{tabular}

ESA= Efecto de sustitución alélica; SESA= Significancia de ESA.

${ }^{1}$ El marcador designado es el gen seguido con la identificación dbSNP en NCBI, posición del SNP de acuerdo al Btau_4.6.1 partir del ensamblado genómico (http://www.ncbi.nlm.nih.gov/SNP/; accedido por última vez el 04/01/2013). 
mente ácido oleico, C18:1, cis-9) sugiere que podría haber una depresión de la actividad de las desaturasas (Chung et al., 2007). Baeza et al. (2012) encontraron efectos significativos de los polimorfismos SRPR sobre la actividad de las desaturasas en novillos Brangus alimentados a pasto, destacando la importancia de los procesos de señalización celular para el transporte y la activación temprana de la proteína, y un papel de apoyo para estos mecanismos en la variabilidad de la composición y perfil de ácidos grasos en la carne bovina. El gen SRPR codifica una subunidad del receptor de reconocimiento de señal de partículas (proteínas de secreción y de membrana) en el retículo endoplásmico.

\section{CONCLUSIONES}

Este trabajo mediante herramientas genómicas ha sido una oportunidad para observar el comportamiento de los diferentes marcadores de las vías metabólicas en estas características evaluadas en el engorde de novillos Hereford. Los SNP no sinónimos

\section{BIBLIOGRAFÍA}

Arranz, J.J.; Coppieters, W.; Berzi, P.; Cambisano, N.; Grisart, B.; Karim, L.; Marcq, F.; Moreau, L.; Mezer C.; Riquet, J.; Simon, P.; Vanmanshoven, P.; Wagenaar, D. and Georges, M. 1998. A QTL affecting milk yield and composition maps to bovine chromosome 20: A confirmation. Anim Genet, 29: 107-115.

Baeza, M.C; Corva, P.M.; Soria, L.A.; Pavan, E.; Rincón, G. and Medrano, J.F. 2012. Genetic variants in a lipid regulatory pathway as potential tolos for improving the nutritional quality of grass-fed beef. Anim Genet, doi:10.111/j.13652052.2012.02386.x

Baeza, M.C.; Corva, P.M.; Soria, L.A.; Rincon, G.; Medrano, J.F.; Pavan, E.; Villarreal, E.L.; Schor, A.; Melucci, L.; Mezzadra, C. and Miquel, M.C. 2011. Genetic markers of body composition and carcass quality in grazing Brangus steers. Genet Mol Res, 10: 3146-3156.

Baldi, F.; Banchero, G.; LaManna, A.; Fernández, (funcionales) en los genes GHR e INSIG1 son recomendados como marcadores candidatos de variación en el nivel de engrasamiento, la puntuación de marmoleado, la terneza de la carne con 21 días de maduración y la relación de ácidos poli-insaturados n-3 y n-6. Estos SNP podrían ser integrados a los paneles de marcadores para la selección y se incrementaría el valor de las características de calidad de carne, composición de la canal, evitando los efectos perjudiciales en la calidad de la carne. Un incremento en las dimensiones del experimento podría permitir estudiar si los resultados preliminares encontrados se mantienen constantes con los diferentes tratamientos nutricionales o si por lo contrario tienen efectos diferenciales.

\section{AGRADECIMIENTOS}

A la Sociedad de Criadores de Hereford de Uruguay y a Tacuarembó-Marfrig por su cooperación a través del Proyecto de Investigación y Desarrollo del INIA: Engorde y Calidad de Carne.

E. y Pérez, E. 2010. Efecto del manejo nutricional post-destete y durante el período de terminación sobre las características de crecimiento y eficiencia de conversión en sistemas de recría y engorde intensivo. Producción de carne desde una invernada de precisión. Serie Actividades de Difusión, INIA. N609.

Bauman, D.E.; Baumgard, L.H.; Corl, B.A. and Griinari, J.M. 1999. Biosynthesis of conjugated linoleic acid in ruminants. Proceedings of the American Society of Animal Science Meeting. Indianapolis, Indiana. pp. 1-5.

Bonanome, A. and Grundy, S.M. 1988. Effect of dietary stearic acid on plasma cholesterol and lipoprotein levels. N Engl J Med, 318: 12441248.

Campbell, E.M.; Gallagher, D.S.; Davis, S.K.; Taylor, J.F. and Smith, S.B. 2001. Rapid communication: mapping of the bovine stearoylcoenzyme $A$ desaturase (SCD) gene to BTA26. J Anim Sci,

Archivos de zootecnia vol. 63, núm. 241, p. 82. 


\section{PANEL SNP EN GENES CANDIDATOS DE VÍAS METABÓLICAS PARA CARNE}

79: $1954-1955$.

Chagas, L.M.; Bass, J.J.; Bleache, D.; Burke, C.R.; Kay, J.K.; Lindsay, D.R.; Lucy, M.C.; Martin, G.B.; Meier, S.; Rhodes, F.M.; Roche, J.R.; Thatcher, W.W. and Webb, R. 2007. Invited review: New perspectives on the roles of nutrition and metabolic priorities in the subfertility of highproducing dairy cows. J Dairy Sci, 90: 40224032.

Chung, K.Y.; Lunt, D.K.; Kawachi, H.; Yano, H. and Smith, S.B. 2007. Lipogenesis and stearoylCoA desaturase gene expression and enzyme activity in adipose tissue of short- and long-fed Angus and Wagyu steers fed corn- or haybased diets. J Anim Sci, 85: 380-387.

Cooke, R.F.; Arthington, J.D.; Araujo, D.B.; Lamb, G.C. and Ealy, A.D. 2008. Effects of supplementation frequency on performance, reproductive, and metabolic responses of Brahmancrossbred females. J Anim Sci, 86: 2296-2309.

Crawford, D.C. and Nickerson, D.A. 2005. Definition and clinical importance of haplotypes. Annu Rev Med, 56: 303-320.

DeAtley, K.L.; Rincón, G.; Farber, C.R.; Medrano, J.F.; Luna-Nervarez, P.; Enns, R.M; VanLeeuwen, D.M.; Silver, G.A. and Thomas, M.G. 2010. Genetic analysis involving microsatellite ETH10 genotypes on bovine chromosome 5 and performance trait measures in Angus and Brahman-influenced cattle. J Anim Sci, 89: 2031-2041.

Dekkers, J.C.M. 2004. Commercial application of marker-and gene-assisted selection in livestock: Strategies and lessons. J Anim Sci, 82(E. Suppl.): E313-E328.

Edens, A. and Talamantes, F. 1998. Alternative processing of growth hormone receptor transcripts. Endocr Rev, 19: 559-582.

Enoch, H.G.; Catala, A. and Strittmatter, P. 1976. Mechanism of rat liver microsomal stearyl-CoA desaturase. Studies of the substrate specificity, enzyme-substrate interactions, and the function of lipid. J Biol Chem, 251: 5095-5103.

Etherton, T.D. 2004. Somatotropic function: The somatomedin hypothesis revisited. J Anim Sci, 82(E. Suppl.): E239-E244.

Folch, J.; Lees, M. and Sloane-Stanley, G.H. 1957. A simple method for the isolation and purification of total lipids from animal tissues. $\mathrm{J}$ Biol Chem, 193: $265-275$.
Fruhbeck, G. 2006. Intracellular signalling pathways activated by leptin. Biochem J, 393: 7-20.

Garrett, A.J.; Rincon, G.; Medrano, J.F.; Elzo, M.A.; Silver, G.A. and Thomas, M.G. 2008. Promoter region of the bovine growth hormone receptor gene: Single nucleotide polymorphism discovery in cattle and association with performance in Brangus bulls. J Anim Sci, 86: 3315-3323.

Ge, W.; Davis, M.E.; Hines, H.C.; Irvin, K.M. and Simmen, R.C. 2003. Association of single nucleotide polymorphisms in the growth hormone and growth hormone receptor genes with blood serum insulin-like growth factor I concentration and growth traits in Angus cattle. J Anim Sci, 81: 641-648.

Ge, W.; Davis, M.E.; Hines, H.C. and Irvin, K.M. 1999. Two-allelic DGGE polymorphism detected in the promoter region of the bovine GHR gene. Anim Genet, 30: 71.

Georges, M.; Nielsen, D.; Mackinnon, M.; Mishra, A.; Okimoto, R.; Pasquino, A.T.; Sargeant, L.S.; Sorensen, A.; Steele, M.R.; Zhao, X.; Womack, J.E.; Hoeschele, I. 1995. Mapping quantitative trait loci controlling milk production in dairy cattle by exploiting progeny testing. Genetics, 139: 907-920.

Hale, C.S.; Herring, W.O.; Shibuya, H.; Lucy, M.C., Lubahn, D.B.; Keisler, D.H. and Johnson, G.S. 2000. Decreased growth in Angus steers with a short TG-microsatellite allele in the $\mathrm{P} 1$ promoter of the growth hormone receptor gene. J Anim Sci, 78: 2099-2104.

Jacobs, A.A.A.; Van Baal, J.; Smits, M.A.; Taweel, H.Z.H.; Hendriks, W.H.; Van Vuuren, A.M. and Dijkstra, J. 2011. Effects of feeding rapeseed oil, soybean oil, or linseed oil on stearoyl-CoA desaturase expression in the mammary gland of dairy cows. J Dairy Sci, 94: 874-87.

Khan, I.A.; Mort, M.; Buckland, P.R.; O'Donovan, M.C.; Cooper, D.N. and Chuzhanova, N.A. 2006. In silico discrimination of single nucleotide polymorphisms and pathological mutations in human gene promoter regions by means of local DNA sequence context and regularity. In Silico Biol, 6: 23-34.

Kromhout, D.; Menotti, A.; Kestleloot, H. and Sans, S. 2002. Prevention of coronary heart disease by diet and lifestyle: Evidence from prospective cross-cultural, cohort, and interventional 
studies. Circulation, 105: 893-898.

Lengi, A.J. and Corl, B.A. 2010. Factors influencing the differentiation of bovine preadipocytes in vitro. J Anim Sci, 88: 1999-2008.

Lengi, A.J. and Corl, B.A. 2007. Identification and characterization of a novel bovine stearoylCoA desaturase isoform with homology to human SCD5. Lipids, 42: 499-508.

Lucy, M.C.; Johnson, G.S; Shibuya, H.; Boyd, C.K. and Herring, W.O. 1998. Rapid communication: Polymorphic (GT)n microsatellite in the bovine somatotropin receptor gene promoter. J Anim Sci, 76: 2209-2210.

Maj, A.; Oprzadek, J.; Dymnicki, E. and Zwierzchowski, L. 2006. Association of the polymorphism in the 5'-noncoding region of the bovine growth hormone receptor gene with meat production traits in polish black-and-white cattle. Meat Sci, 72: 539-544.

Maj, A.; Oprzadek, J.; Oprzadek, A.; Dymnicki, E. and Zwierzchowski, L. 2004. Polymorphism in the 5 -noncoding region of the bovine growth hormone receptor gene and its association with meat production traits in cattle. Anim Res, 53: 503-514.

Mauvoisin, D. and Mounier, C. 2011. Hormonal and nutritional regulation of SCD1 gene expression. Biochimie, 93: 78-86.

Orrù, L.; Cifuni, G.F.; Piasentier, E.; Corazzin, M.; Bovolenta, S. and Moioli, B. 2011. Association analyses of single nucleotide polymorphisms in the LEP and SCD1 genes on the fatty acid profile of muscle fat in Simmental bulls. Meat Sci, 87: 344-348.

Paton, C.M. and Ntambi, J.M. 2009. Biochemical and physiological function of stearoyl-CoA desaturases. Amer J Physiol-Endocrinol Metabol, 297: 28-37.

Rawlings, J.S.; Rosler, K.M. and Harrison, D.A. 2004. The JAK/STAT signaling pathway. J Cell Sci, 117: 1281-1283.

Rincón, G.; Islas-Trejo, A.; Castillo, A.R.; Bauman, D.E.; German, B.J. and Medrano, J.F. 2012. Polymophisms in gene in the SREBP-1 signalling and SCD are associated with milk fatty acid composition in Holstein cattle. J Dairy Res 79:
66-75.

Rincón, G.; Farber, E.A.; Farber, C.R.; Nkrumah, J.D. and Medrano, J.F. 2009. Polymorphisms in the STAT6 gene and their association with carcass traits in feedlot cattle. Anim Genet, 40: 878-882.

Ron, M. and Weller, J.I. 2007. From QTL to QTN identification in livestock - Winning by points rather than knock-out: A review. Anim Genet, 38: 429-439.

Sambrock, J.; Fritsch, E.F. and Maniatis, T. 1989. Molecular Cloning: A Laboratory Manual. Cold Springer Harbor Laboratory Press. New York.

SAS. 2007. User's Guide: Statistics. Version 6.14 Edition 2007. SAS Inst. Inc. Cary. NC.

Schindler, C. and Plumlee, C. 2008. Interferons pen the JAK-STAT pathway. Semin Cell Dev Biol, 19: 311-318.

Schmid, A.; Collomb, M.; Sieber, R. and Bee, G. 2006. Conjugated linoleic acid in meat products: A review. Meat Sci, 73: 29-41.

Sherman, E. L.; Nkrumah, J.D; Murdoch, B.M; Li, C.; Wang, Z.; Fu, A. and Moore, S.S. 2008. Polymorphisms and haplotypes in the bovine neuropeptide $\mathrm{Y}$, growth hormone receptor, ghrelin, insulin-like growth factor 2, and uncoupling proteins 2 and 3 genes and their associations with measures of growth, performance, feed efficiency, and carcass merit in beef cattle. J Anim Sci, 86: 1-16.

Taniguchi, M.; Utsugi, T.; Oyama, K.; Mannen, H.; Kobayashi, M.; Tanabe, Y.; Ogino, A. and Tsuji, S. 2004. Genotype of stearoyl-CoA desaturase is associated with fatty acids composition in Japanese Black cattle. Mamm Genome, 14: 142-148.

Zhang, Q.; Boichard, D.; Hoeschele, I.; Ernst, C.; Eggen, A.; Murkve, B.; Pfister-Genskow, M.; Witte, L.A.; Grignola, F.E.; Uimari, P.; Thaller, G. and Bishop, M.D. 1998. Mapping quantitative trait loci for milk production and health of dairy cattle in a large outbred pedigree. Genetics, 149: 1959-1973.

Zhu, T.; Goh, E.L.; Graichen, R.; Ling, L. and Lobie, P.E. 2001. Signal transduction via the growth hormone receptor. Cell Signal, 13: 599-616.

Archivos de zootecnia vol. 63, núm. 241, p. 84. 